\title{
Simulation of 3D-Microstructure in Free-cutting Steel 9SMn28 under Water Cooling Condition with Convection and Porosity
}

\author{
Jinlong WANG ${ }^{11}$ Fuming WANG, ${ }^{1)}$ Changrong $\mathrm{LI}^{21}$ and Jiongming ZHANG ${ }^{1)}$ \\ 1) School of Metallurgical and Ecological Engineering, University of Science and Technology Beijing, Beijing 100083, China. \\ E-mail: wangjlong78@126.com $\quad$ 2) School of Materials Science and Engineering, University of Science and Technology \\ Beijing, Beijing 100083, China.
}

(Received on July 9, 2009; accepted on November 9, 2009)

\begin{abstract}
The 3D-microstructure in 9SMn28 free-cutting steel under water cooling condition was simulated based on the finite element-cellular automaton method with convection and porosity, and the simulated results are consistent with that of the experiment. The casting of 9SMn28 alloy is a stepwise solidification mode under water cooling condition. Convection is mainly made of buoyancy-driven flows and feeding flows in perpendicular direction of the solid-liquid interface. Convection is the reason of shaggy on free surface; it can decrease pipe shrinkage and columnar grain zone. Flow field plays an importance role in even temperature field and irregulation porosity morphology. The simulated result of pipe shrinkage is consistent with that of this experiment; the simulated result of porosity is identical with that of this experiment basically.
\end{abstract}

KEY WORDS: microstructure; convection; porosity; finite element-cellular automaton model.

\section{Introduction}

Casting is a key manufacturing process. Hence much work has been done on computer modeling of solidification experiments. Modeling microstructure, both physically and numerically, is especially useful in order to understand better its fundamental changes in metals cast under specific conditions. In simulation, it is necessary to consider some phenomena during solidification processes, such as convection and porosity.

Melt convection plays an important role in the solidification of metal castings. Different macrosegregation patterns are formed or at least strongly influenced by the melt flow and grain size. Usually, solute elements distribute more evenly in the smaller grains. Convection is caused by solidification itself in most solidification processes. Furthermore, convection is usually unsteady and of a long-range nature, and can thus change the environment in which the microstructure grows. Convection causes the macroscopic movement of the liquid phase, its penetration into the slurry zone and transport of solutes in the direction of convection flows. In addition, the convection flows pick up the freefloating, solute-lean solid grains and transport them within the slurry region until the moment when these grains become heavy enough to settle down. ${ }^{1)}$ Coupled convective and morphological instabilities during directional solidification of an alloy have also been simulated using the phasefield method..$^{2-4)}$ Cellular automaton (CA) techniques have also become available. ${ }^{5,6)}$ Porosity is one of the major defects in castings, which results in a decrease of the mechanical properties, in particular the fatigue and ultimate tensile strengths. It is induced by two mechanisms, solidification shrinkage and gas segregation, which occur concomitantly but with different intensities. Figure 1 shows schematics of a simplified casting. Solidification induces three types of voids: (i) at a free surface (e.g., of risers), the level of liquid decreases as solidification proceeds (piping); (ii) within closed liquid pockets (hot spots), a macropore surrounded by microporosity will be present; (iii) microporosity finally dispersed within the mushy zone might finally appear at an early stage of solidification (gas porosity) or deep in the mushy zone, especially when a dense interdendritic phase forms (shrinkage porosity). ${ }^{7}$ Therefore, it is of actual significance to simulate the metal microstructure with convection and porosity.

Up to now, such calculations, however, have seldom been reported. Therefore, the aim of this paper is to simulate microstructure during the metal solidification with convection and porosity based on cellular automaton model and finite

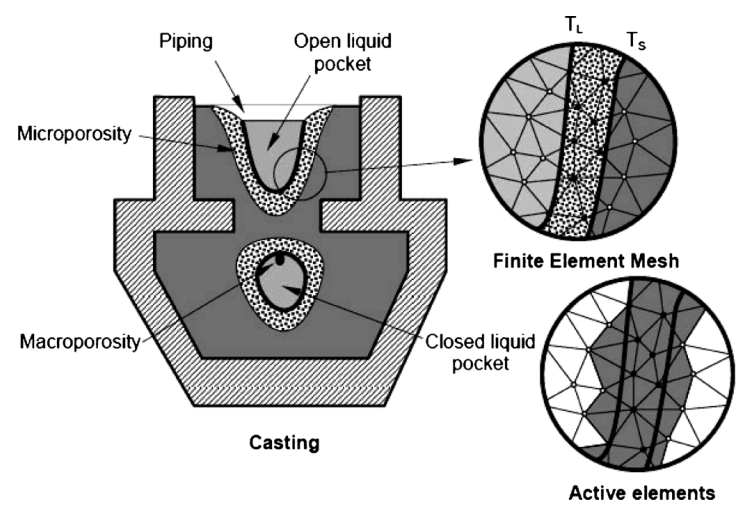

Fig. 1. Schematics of a casting showing piping at a free surface and macroporosity formation in a closed liquid pocket. ${ }^{7)}$ 
element (FE) method which are combined to build macromicro FE-CA coupling model, that is, the CAFE method, and the results are validated through experiments. This model first calculates heat transfer equation of castings during solidification processes using FE model, and then sets up regulation of nucleation and growth of cells through mechanism of the formation of microstructure, the calculated results of FE model are coupled to CA model to simulate microstructure. In 1990s, Rappaz and Gandin, ${ }^{8,9)}$ Nastac and Stefanescu, ${ }^{10)}$ and $\mathrm{Zhu}{ }^{11,12)}$ successively simulated the metal microstructure during solidification processes by this model.

\section{Mathematical Physics Model of the CAFE}

\subsection{Thermo-physical Property Calculation}

An extensive database for the calculation of thermophysical properties has been developed which utilizes the phase fraction information predicted with the minimization routines developed by Lukas et al., ${ }^{13)}$ and extended by Kattner et al. ${ }^{14)}$ These properties include density, specific heat, enthalpy, latent heat, thermal conductivity, and liquid viscosity, and so on. The thermodynamic calculation is based on the thermodynamic database from CompuTherm LLC. A simple pair-wise mixture model which is similar to that used to model thermodynamic excess functions in multicomponent alloys is used to calculate the properties. ${ }^{15)}$

$$
P=\sum x_{i} P_{i}+\sum_{i} \sum_{j \succ i} x_{i} x_{j} \sum_{v} \Omega_{v}\left(x_{i}-x_{j}\right)^{v}
$$

Where $P$ is the phase property, $P_{i}$ is the property of the pure element in the phase, $\Omega_{i}$ is a binary interaction parameter, and $x_{i}$ and $x_{j}$ are the mole fractions of elements $i$ and $j$ in that phase.

\subsection{Phase Transformation}

\subsubsection{Enthalpy-Temperature ${ }^{16)}$}

The temperature field obeys the nonstationary heat flow equation:

$$
\operatorname{div}(k(x, T) \operatorname{grad} T(x, t))=c_{p}(x, t) \frac{\partial T(x, t)}{\partial t}-L \frac{\partial f_{s}(x, t)}{\partial t}
$$

Where $k$ is thermal conductivity, $c_{p}$ is the specific heat capacity, $L$ is the latent heat of fusion, and $T$ and $f_{s}$ are the temperature and solid fraction fields, respectively.

The term on the right-hand side of Eq. (2) represents the variation in enthalpy at a given point of the casting, the enthalpy $H$ being defined as:

$$
H(T)=\int_{0}^{T} c_{p}(T) d T+L\left(1-f_{s}\right)
$$

In a more general approach, the enthalpy also can be a function of time, cooling rate, grain density, etc. However, for a given heat flow, the Eq. (2) states that the variation in enthalpy is independent of the solidification path. Therefore, it might be advantageous at the macroscopic scale to choose enthalpy as the variable rather than temperature.
Then Eq. (2) then can write:

$$
\operatorname{div}(k(x, T(H(x, t))) \operatorname{grad} T(H(x, t)))=\frac{\partial H(x, t)}{\partial t} \ldots
$$

\subsubsection{Phase Diagram ${ }^{17)}$}

It will be assumed that the alloy, which will be solidified, is a binary alloy. The equilibrium phase diagram of the binary alloy is linearized. Due to the linearization, the liquidus may be assumed to be a straight line with slope $m$. The solidus also being linear, the partition coefficient $k_{c}$ can be defined as: $c_{s}^{*}=k_{c} c_{l}^{*}$. Where $c_{s}^{*}$ and $c_{l}^{*}$ are the equilibrium concentrations of the solid and the liquid for an arbitrary temperature $T_{0}$. Moreover, denote by $T_{m}$ the melting point of the pure substance. Within the mushy zone, above the eutectic temperature $T_{\text {eut }}$, the temperature $T$ can be related to the equilibrium concentration of the liquid $c_{l}^{*}$ by $T=T_{m}+m c_{l}^{*}$. When the eutectic temperature is reached, $T=T_{\text {eut }}$ and $c_{l}^{*}=c_{\text {eut }}$ are fixed by the phase diagram and the average enthalpy $H$ becomes a linear function of the solid fraction $f_{s}$.

\subsection{Fluid Flow}

\subsubsection{The Mushy Zone as a Porous Medium}

When neglecting grain-movement and thus the solid phase being fixed, the mushy zone can be viewed as a skeleton of dendrites where the liquid metal flows through. This situation may be modeled by flow through porous media. ${ }^{18,19)}$ Such flow is governed by Darcy's law, which states that the average velocity $v$ is linear with respect to the pressure gradient $\nabla p$. For the situation where the solid is fixed, we have $v=v_{l} f_{l}$. In order to include and study the effects of gravity on interdendritic flow, a gravity term is added to Darcy's law, which leads to

$$
v_{l} f_{l}=-\frac{K}{\mu}\left(\nabla p-\rho_{l} g\right)
$$

Where $K$ is the permeability conductivity, $\mu$ the viscosity, $g$ gravity, $\rho_{l}$ the liquid density, $f_{l}$ represents the liquid fraction. $^{20)}$

Using a thermal expansion coefficient, $\beta_{T}=(1 / V)$. $(\partial V / \partial T)=-(1 / \rho) \cdot(\partial \rho / \partial T)$, and a solutal expansion coefficient, $\beta_{c}=(1 / V) \cdot\left(\partial V / \partial c_{l}\right)=-(1 / \rho) \cdot\left(\partial \rho / \partial c_{l}\right)$, the well known Boussinesq approximation states that, for fixed reference values $T_{\text {ref }}, c_{\text {ref }}$ for temperature and concentration, respectively, and with $\rho_{0}=\rho\left(T_{\text {ref }}, c_{\text {ref }}\right)$, the following equation is applied. ${ }^{17)}$

$$
\rho_{l}=\rho_{0}\left(1-\beta_{T}\left(T-T_{\text {ref }}\right)-\beta_{C}\left(c_{1}-c_{\text {ref }}\right)\right)
$$

where $V$ is a reference volume, $\rho$ the density, and $c_{l}$ the concentration of solute within the liquid. This corresponds to a linearization of $\rho_{l}$ as a function of $T$ and $c_{l}$ around $\left(T_{\text {ref }}, c_{\text {ref }}\right)$.

\subsubsection{Permeability}

Note that interdendritic fluid flow depends considerably on the permeability parameter, which is itself a function of the packing of the dendrites (thus of the solid fraction) and their orientation. The permeability is expressed as a func- 
tion of the specific surface of the porous medium. If $S_{0}$ designates the specific surface, defined as the solid surface exposed to the fluid per unit volume of solid, then, the well known Carman-Kozeny relationship reads ${ }^{17)}$.

$$
K=\frac{1}{5 S_{0}^{2}} \frac{f_{l}^{3}}{\left(1-f_{l}\right)^{2}}
$$

Note that the reliability of permeability models is significantly limited by the lack of permeability data. Whereas the above model has been validated experimentally for higher solid fractions (e.g., Ref. 21)), no data is available for the regions with small solid fraction because of difficulties associated with their measurement.

\subsubsection{Viscosity}

The aim of the permeability approach described above is to modify the Navier-Stokes equations by adding a permeability term such that, for small liquid fractions $f_{l}$ (and thus small permeability), the fluid flow equations are approximating Darcy's law, whereas, for $f_{l}=1$, they remain the usual Navier-Stokes equations, which model the fluid flow within the completely liquid region. If the permeability tends towards zero, the velocity shall also be forced to be zero, as the solid is supposed to be fixed.

\subsection{Model of the CA}

\subsubsection{Heterogeneous Nucleation}

A continuous nucleation distribution function, $d n / d(\Delta T)$, can be used to describe the grain density change, $d n$, which is induced by an increase in the undercooling, $d(\Delta T)$. The distribution function $d n / d(\Delta T)$ is described by the equation $^{8)}$

$$
\frac{d n}{d(\Delta T)}=\frac{n_{\max }}{\sqrt{2 \pi} \Delta T_{\sigma}} \exp \left[-\frac{1}{2}\left(\frac{\Delta T-\Delta T_{\max }}{\Delta T_{\sigma}}\right)\right] \ldots
$$

Where $\Delta T$ is the calculated local undercooling, $\Delta T_{\max }$ is the mean undercooling, $\Delta T_{\sigma}$ is the standard deviation, $n_{\max }$ is the maximum nucleation density which can be reached when all the nucleation sites are activated while cooling.

\subsubsection{Dendrite Tip Growth Kinetics}

In castings, the growth kinetics of both columnar and equiaxed morphologies can be calculated with the aid of the KGT (Kurz, Givoanola, and Trivedi) model. ${ }^{22)}$ In the actual simulation process, in order to accelerate the course of computation, KGT model is fitted and the following equation is gained.

$$
v(\Delta T)=a_{2} \Delta T^{2}+a_{3} \Delta T^{3}
$$

Where $a_{2}$ and $a_{3}$ are the coefficients of the multinomial of dendrite tip growth velocity, $\Delta T$ is the total undercooling of the dendrite tip.

\subsubsection{Coupling the $\mathrm{FE}$ and $\mathrm{CA}^{9)}$}

The aim of this work is to combine the FE and CA calculations in a single model to predict simultaneously the microstructure development as a function of the thermal field and the influence of the latent heat release of the grains on the calculated thermal history. Within each time step, the known temperature at time $t, T_{v}^{t}$, and the volumetric enthalpy variation, $\delta H_{v}$, of the cell $v$ are first interpolated by using the linear interpolation coefficients $\phi_{v n}$

$$
\begin{gathered}
T_{v}^{t}=\sum_{n} \phi_{v n} \cdot T_{n}^{t} \\
\delta H_{v}=\sum_{n} \phi_{v n} \cdot \delta H_{n}
\end{gathered}
$$

where the summation over the index $(n)$ is carried out over all the nodal points of the finite element $(I)$ which contains the cell $(v)$. For each cell, the unknown temperature, $T_{v}^{t+\delta t}$, and internal volume fraction of solid, $f_{s, v}^{t+\delta t}$ must satisfy the following relationship

$$
\begin{aligned}
\delta H_{v} & =\rho c_{p} \delta T_{v}-L_{f} \delta f_{s, v} \\
& =\rho c_{p}\left(T_{v}^{t+\delta t}-T_{v}^{t}\right)-L_{f}\left(f_{s, v}^{t+\delta t}-f_{s, v}^{t}\right) \cdots \cdots
\end{aligned}
$$

where $L_{f}$ is the latent heat of fusion per unit volume. Three cases can be encountered for any cell:

(i) No variation of the volume fraction of solid

The explicit temperature is above the liquidus of the alloy (or below the final eutectic temperature) in which case the volume fraction of solid of the cell does not evolve during the time step.

(ii) Solidification of an already mushy cell

The cell has an index $\neq 0$ (no liquid state) and $T_{L}>$ $T_{v}^{t}>T_{\text {eut }}$, the volume fraction of solid in the cell is assumed to follow the microsegregation model of Scheil. Deriving the Scheil equation simply gives

$$
\delta f_{s, v}=\frac{-\delta H_{v}}{\rho c_{p}\left(T_{L}-T_{m}\right)\left(k_{c}-1\right)\left[1-f_{s, v}^{t}\right]^{(k-2)}+L_{f}}
$$

If the cell temperature is equal to the eutectic temperature with a solid fraction lower than unity, $f_{s, v}^{t}<1$, a simple isothermal transformation is made according to Eq. (12) until $f_{s, v}^{t}=1$.

(iii) Capture or nucleation of a cell which was liquid

The cell has its index changed from zero to a positive integer number during the time step. The whole cell is attributed a volume fraction of solid given by the Scheil model at the corresponding explicit temperature, i.e.

$$
\delta f_{s, v}=1-\left[\frac{T_{v}^{t}-T_{m}}{T_{L}-T_{m}}\right]^{1 /\left(k_{c}-1\right)}
$$

The latent heat contributions of all the cells associated with point (i-iii) above are then fed back into the nodal points of the FE mesh according to

$$
\delta f_{s, n}=\frac{\sum_{v} \phi_{v n} \delta f_{s, v}}{\sum_{v} \phi_{v n}}
$$

where the summation is now carried out over all the cell be- 
longing to the elements which have the nodal point, $n$, as a vertex. The denominator appearing in Eq. (15) is a normalization factor. Once the $\delta f_{s, n}$ are known, the new temperatures at the nodal points can be calculated according to

$$
\begin{aligned}
\delta H_{v} & =\rho c_{p} \delta T_{n}-L_{f} \delta f_{s, n} \\
& =\rho c_{p}\left(T_{n}^{t+\delta t}-T_{n}^{t}\right)-L_{f}\left(f_{s, n}^{t+\delta t}-f_{s, n}^{t}\right)
\end{aligned}
$$

\subsubsection{Calculation Method of the CA Model}

New nucleation is formed randomly in the bulk volume as the result of the fall of local temperature of nucleating cell below the critical temperature in the given time step. Crystallographic orientation of crystal growth is randomly chosen from predefined orientation classes. ${ }^{23)}$ The crystallographic orientation $\langle 100\rangle$ is selected preferentially. The growth of grains is realized by capturing the neighboring cells which had the same crystallographic orientation with matrix cell, and this calculation method can realize the competition of crystal growth. ${ }^{24)}$

\subsubsection{Governing Equation}

Momentum conservation equation:

$$
\begin{aligned}
\frac{\rho}{f_{l}} \frac{\partial u}{\partial t} & +\frac{\rho}{f_{l}^{2}}\left(u \frac{\partial u}{\partial x}+v \frac{\partial u}{\partial y}+w \frac{\partial u}{\partial z}\right) \\
= & -\frac{\partial P}{\partial x}+\rho g_{x}+\frac{\partial}{\partial x}\left(\frac{u}{f_{l}} \frac{\partial u}{\partial x}\right)+\frac{\partial}{\partial y}\left(\frac{u}{f_{l}} \frac{\partial u}{\partial y}\right) \\
& +\frac{\partial}{\partial z}\left(\frac{u}{f_{l}} \frac{\partial u}{\partial z}\right)-\left(\frac{\mu}{K}\right) u
\end{aligned}
$$

Mass conservation equation:

$$
\frac{\partial \rho}{\partial t}+\frac{\partial(\rho u)}{\partial x}+\frac{\partial(\rho v)}{\partial y}+\frac{\partial(\rho w)}{\partial z}=0
$$

Energy conservation equation:

$$
\begin{gathered}
\rho \frac{\partial H}{\partial t}+\rho \frac{\partial H}{\partial T}\left(u \frac{\partial T}{\partial x}+v \frac{\partial T}{\partial y}+w \frac{\partial T}{\partial z}\right) \\
=\frac{\partial}{\partial x}\left(k \frac{\partial T}{\partial x}\right)+\frac{\partial}{\partial y}\left(k \frac{\partial T}{\partial y}\right)+\frac{\partial}{\partial z}\left(k \frac{\partial T}{\partial z}\right)
\end{gathered}
$$

$u, v, w$ : velocity vector in $x, y$ and $z$ direction, respectively, $\mathrm{m} / \mathrm{s} ; p$ : pressure, $\mathrm{Pa} ; g_{x}$ : gravity in $x$ direction, $\mathrm{m} / \mathrm{s}^{2} ; t$ : time, $s$.

\section{Simulation of 3D-microstructure in Free-cutting Steel 9SMn28 with Convection and Porosity}

\subsection{Selection of Simulation Parameters}

Figure 2 shows the simulated casting made of 9SMn28 free-cutting steel. All results shown in this paper are the casting center surface in height direction (gravity direction). The casting material is gained by rolled 9SMn28 free-cutting steel; it is melted by vacuum induction furnace in the protection of argon after getting rid of oxide skins. The casting is molded by die casting. Molten steel pouring temperature is $T=1833 \mathrm{~K}$. The top of the casting is air cooling, the others are water cooling. The simulative and experimental conditions of the casting are the same in this paper. In the simulation, the mould is filled with the melt instantaneously. The simulations have been carried out using Procast software.

The calculated results of the maximum nucleation density are approximately $n_{\mathrm{v}, \max }=1 \times 10^{11} \mathrm{~m}^{-3}, n_{\mathrm{s}, \max }=1 \times$ $10^{9} \mathrm{~m}^{-2}$ according to metallographs of the casting in Fig. 3 and calculation method commended by ASTM standard. The other parameters of Gaussian distribution are $\Delta T_{\mathrm{v}, \max }=6 \mathrm{~K}, \Delta T_{\mathrm{v}, \sigma}=2 \mathrm{~K}, \Delta T_{\mathrm{s}, \max }=5 \mathrm{~K}$, and $\Delta T_{\mathrm{s}, \sigma}=1 \mathrm{~K}$ (in the bulk volume indexed as " $\mathrm{v}$ " and at the surface of the mould indexed as "s").

In this work, 9SMn28 alloy has been decomposed into 5 $\mathrm{Fe}-\mathrm{X}$ binary alloy systems, which are $\mathrm{Fe}-\mathrm{C}, \mathrm{Fe}-\mathrm{S}, \mathrm{Fe}-\mathrm{P}$, $\mathrm{Fe}-\mathrm{Mn}$, and $\mathrm{Fe}-\mathrm{Si}$ alloys. The calculated values of liquidus and solidus temperature of 9SMn28 alloy are $T_{L}=1796 \mathrm{~K}$ and $T_{S}=1762 \mathrm{~K}$, respectively. Gibbs-Thompson coefficient is $\Gamma=3 \times 10^{-7} \mathrm{~m} \cdot \mathrm{K}$. The coefficients of dendrite tip growth kinetics are calculated to be $a_{2}=0$ and $a_{3}=7.368 \times$ $10^{-6} \mathrm{~m} \cdot \mathrm{s}^{-1} \cdot \mathrm{K}^{-3}$ based on the data of $T_{L}, \Gamma$ and Composi-

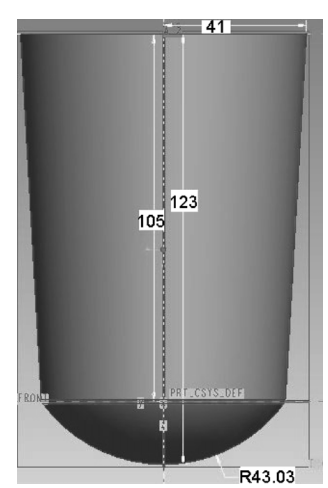

Fig. 2. The simulated casting.

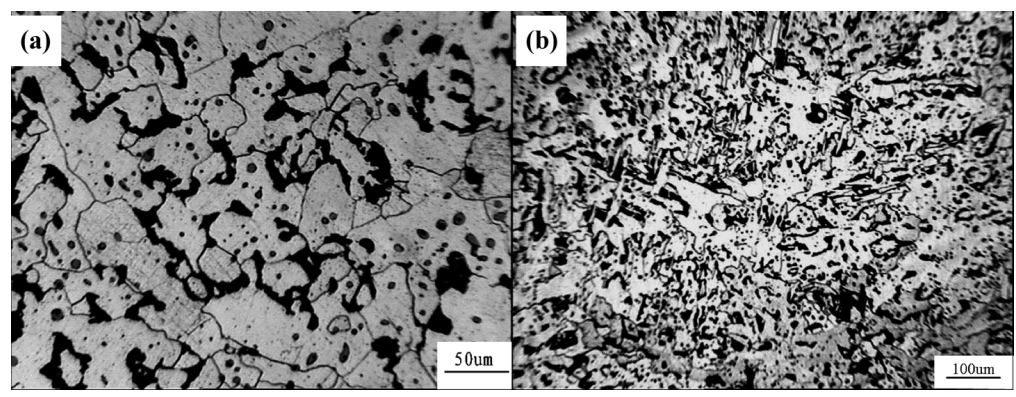

Fig. 3. Metallographs of the casting, (a) equiaxed grains, (b) columnar grains. 
tion $c_{0}$, partition coefficient $k_{c}$, liquidus slope $m$, and solute diffusion coefficient $D_{1}$ of Fe-X alloys (Table 1).

\subsection{The Simulated Temperature Field Results and Discussion}

The simulated results of temperature fields and solidification process are symmetrical. So, they are coupled in a figure (Fig. 4). Figure 5 shows temperature curves and solid curves at locations of A, B, C, D, and E in the Fig. 4(a).

The start of cooling immediately establishes a symmetrical and positive temperature gradient towards the casting center. As soon as temperature drops below the liquidus temperature, the melt is undercooled, and nucleation and solidification begin. This occurs first in the corners, then along the mold walls, and finally in the bulk melt. A significant feature at the initial stages is that the isotherm and the solid fraction isolines are almost identical and very symmetrical, and that they move gradually from corners and casting surface regions towards the casting center, showing that the solidification process is initially controlled by heat extraction merely. So, nucleation and growth are only con- trolled by thermal transmission. These simulated results are same as those of the reference. ${ }^{25)}$ It can be seen from Fig. 4 that undercooling only occurs near solidification interface in which the liquid is first undercooled in order to create conditions for growth of grains. Conducting heat in the melt, temperature of the melt decreases ceaseless, the undercooling zones are enlarged, the undercooling is increased, and the growth zones of nucleation are also enhanced with the development of solidification. Crystal temperature interval of 9SMn28 alloy is small, the temperature decreases of solidification zones are great, and solidification zones are narrow. Solidification layers also increase until casting center step by step. Thereby, the casting of 9SMn28 alloy is a stepwise solidification mode under water cooling condition.

It can be found from solid curves and temperature curves of Fig. 5 that the cooling of surface layer of the casting (at location of E) is quicker, crystal begins in bigger undercooling. Moreover, the release of latent heat of crystallization is always less than the thermal release. Therefore, the solidification occurs in the processes of continuous fall temperature, temperature rapidly decreases after the finish

Table 1. Composition, partition coefficient, liquidus slope, and solute diffusion coefficient of Fe-X alloys.

\begin{tabular}{ccccc}
\hline Element & $c_{0}$, mass $\%$ & $m, \mathrm{~K}$ & $k_{c}$ & $D_{l}, \mathrm{~m}^{2} / \mathrm{s}$ \\
\hline $\mathrm{C}$ & 0.12 & -58 & 0.17 & $11 \times 10^{-9}$ \\
$\mathrm{Mn}$ & 1.08 & -5 & 0.68 & $2.4 \times 10^{-9}$ \\
$\mathrm{~S}$ & 0.30 & -21 & 0.05 & $3.5 \times 10^{-9}$ \\
$\mathrm{P}$ & 0.054 & -48.3 & 0.13 & $4.6 \times 10^{-9}$ \\
$\mathrm{Si}$ & 0.016 & -18.7 & 0.65 & $8.5 \times 10^{-9}$ \\
\hline
\end{tabular}
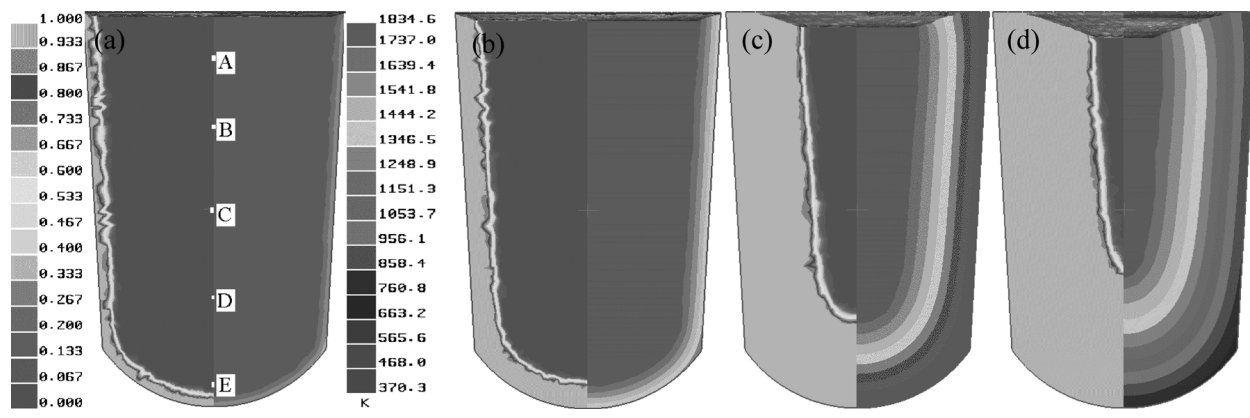

Fig. 4. The simulated temperature field (on the right) and solidification process (on the left) results of the casting ((a) $3.92 \mathrm{~s}$, (b) $9.20 \mathrm{~s}$, (c) $39.07 \mathrm{~s}$ and (d) $53.77 \mathrm{~s}$ ). The left and right markings of the Fig. 4(a) are fraction of solid and temperature, respectively.
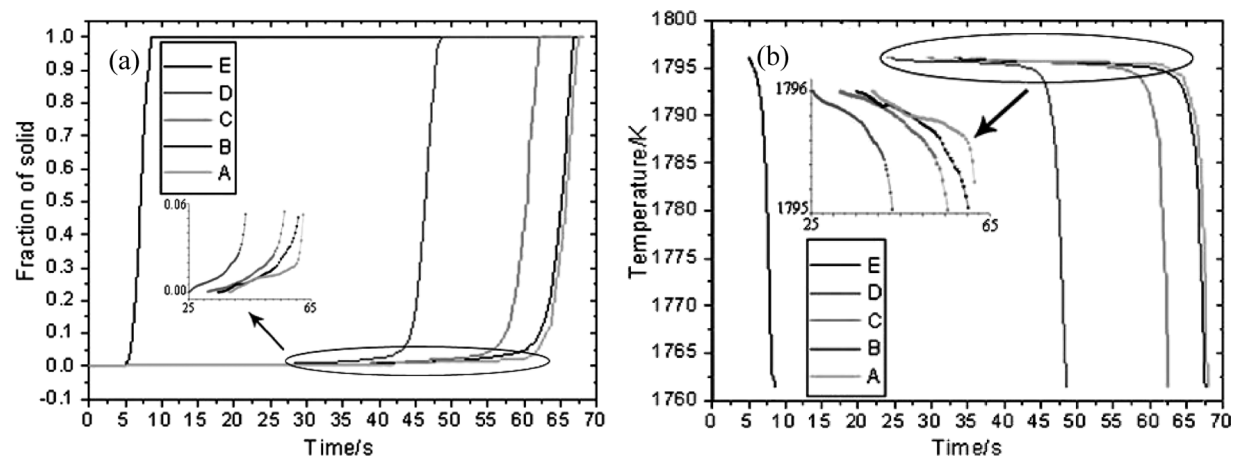

Fig. 5. Solid curves (a) and temperature curves (b) from $T_{S}$ to $T_{L}$ at locations of A, B, C, D and E. 


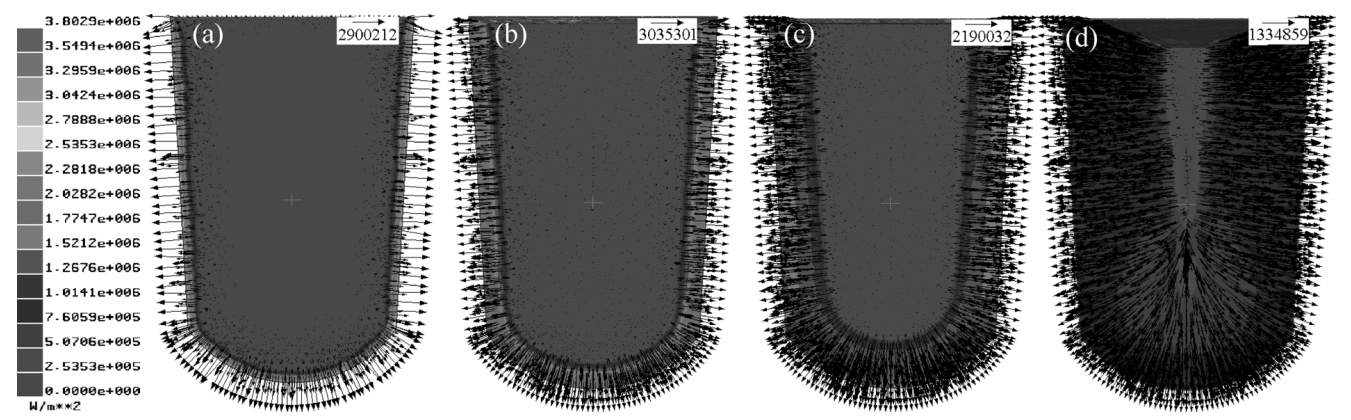

Fig. 6. The calculation results of heat flux ((a) $0 \mathrm{~s}$, (b) $8.24 \mathrm{~s}$, (c) $20.04 \mathrm{~s}$ and (d) $58.77 \mathrm{~s}$ ), the left marking is heat flux, top right corner is heat flux vector and heat flux, and their units are $\mathrm{W} / \mathrm{m}^{2}$.
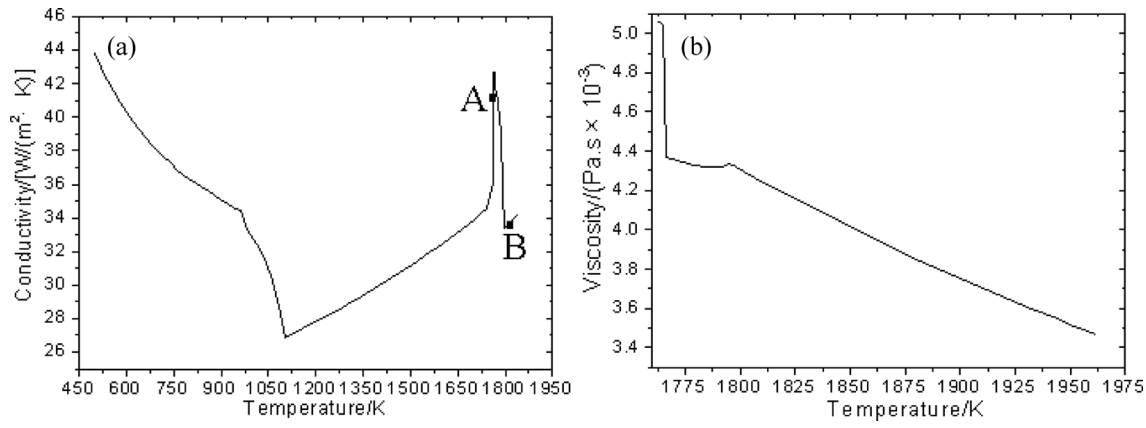

Fig. 7. Conductivity (a) and viscosity (b) of $9 \mathrm{SMn} 28$ alloy (A and B are $T_{S}$ and $T_{L}$, respectively).

of solidification. In the casting interiors, heat flux decreases gradually. The casting interiors (at locations of B, C, and D) are near-equilibrium cooling at the initial stages of solidification. Temperature keeps invariableness basically because the release of latent heat of crystallization is general equal to the thermal release. With the progress of solidification, the release of latent heat of crystallization is less than the thermal release. Thus, temperature falls, and the gradient of temperature is greater. The location of $\mathrm{A}$ is also surface layer of the casting, but its thermal release is slower because the top of the casting is air cooling. Cooling and solidification modes of the location of $\mathrm{A}$ resemble the locations of B, C, and D.

The solid velocity of bottom parts of the casting is quicker than top parts because of the different of cooling condition and the influence of convection. It can be found from Fig. 5 that solid time at locations from A to E, namely from top to bottom of the casting, enhances gradually. Whole process of solidification at location $\mathrm{E}$ is quicker. Solidification at the initial stages is slower at locations A, B, $\mathrm{C}$, and $\mathrm{D}$, while their solid velocities are almost identical with location E after the initial stages. Applying water cooling, the outer surface of the casting is rapidly cooled during solidification processes, whereas the variety of the casting center is slower, the temperature gradient from centre to surface increases gradually. Moreover, the variety range of temperature is great; the isothermal zone is narrow.

\subsection{The Calculated Heat Flux Results and Discussion}

At the beginning of cooling, heat flux of surface and bottom of the casting which are water cooling is more than the top which is air cooling (Fig. 6(a)). Because of the increase of thickness and the lessening of difference in temperature, heat flux is decreased from surface to center of the casting gradually. Distributing maps of heat flux resemble distribut- ing maps of temperature. When solidification starts, heat flux increases due to phase transformation (Fig. 6(b)). Because of the increase of thickness, the lessening of difference in temperature, and the change of conductivity in solidification zones (Fig. 7(a)), heat flux decreases with the development of solidification (Fig. 6(c)). In final stages, conductivity of solidification zone exteriors increases, while that of interiors is lower due to distribution of temperature, and these weaken heat transmission in solidification zones. Moreover, thickness of solidification zone is heightened continuously. So, heat flux is reduced gradually (Fig. 6(d)).

\subsection{The Simulated Flow Field Results and Discussion}

Figures 8, 9, and $\mathbf{1 0}$ show the simulated results of flow fields, temperature maps and flow field maps, and temperature fields of no liquid flow, respectively. Figure 11 shows flow velocity curves at locations of $\mathrm{A}, \mathrm{B}, \mathrm{C}, \mathrm{D}$, and $\mathrm{E}$ in the Fig. 4(a).

The melt is Newtonian fluid and can not be compressed, the melt viscosity of 9SMn28 alloy increases with temperature decrease (Fig. 7(b)). Buoyancy-driven melt convection comes into being due to gravity; feeding flows in perpendicular direction of the solid-liquid interface are formed by solidification shrinkage. A flow field is formed when cooling starts, and it is symmetrical (Fig. 8(a)). With the development of solidification, the flow fields of part circulations in top and bottom of the casting and whole circulation are formed by the effects of buoyancy-driven melt convection and feeding flows, they can be seen from Fig. 8(b). There is velocity vector upwards on top surface of the casting as a result of ceaseless enhancement of liquid flows in Figs. 8(b) and $8(\mathrm{c})$; the melt takes on a surge phenomenon, it is mostly the reason of shaggy on top surface. Flow velocity of the melt is reduced due to continuous increase of melt 


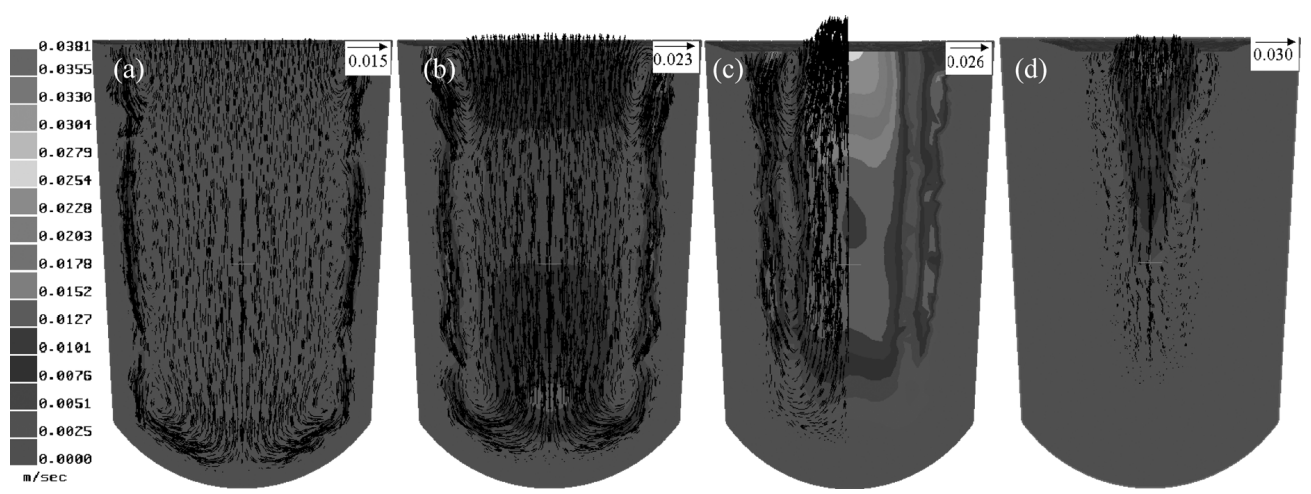

Fig. 8. The simulated flow field results of the casting ((a) $2.40 \mathrm{~s}$, (b) $5.59 \mathrm{~s}$, (c) $9.94 \mathrm{~s}$ and (d) $32.20 \mathrm{~s}$ ), the left marking is flow velocity, top right corner is flow velocity vector and flow velocity, and their units are $\mathrm{m} / \mathrm{s}$.
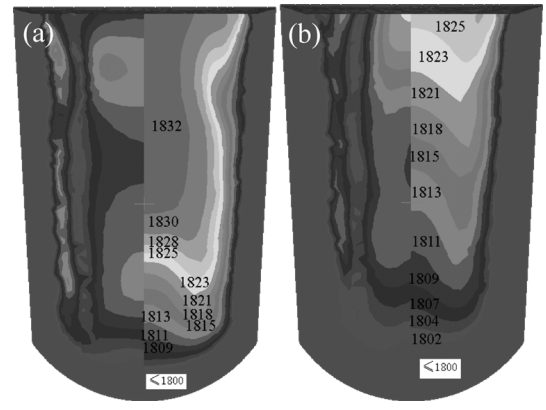

Fig. 9. Temperature maps (right) and flow field maps (left) ((a) $5.59 \mathrm{~s}$ and (b) $9.94 \mathrm{~s})$.
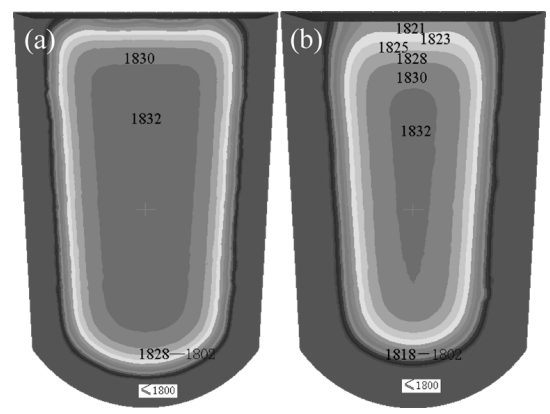

Fig. 10. Temperature fields of no liquid flow ((a) $5.62 \mathrm{~s}$ and (b) $9.88 \mathrm{~s})$.

viscosity with the unceasing decrease of temperature. It cannot form the flow fields of part circulations in the bottom of the casting because the melt viscosity near solidification parts increases, and it weakens feeding flows, it can be seen from Fig. 8(c). The degree of weakened feeding flows on the top is less. So, there is still the flow field of part circulation (Fig. 8(c)). With continuous development of solidification, the circulation of flow field can not be formed (Fig. 8(d)). Liquid flows are blocked by continuous increase of melt viscosity at final stages of solidification. So, there is not flowing occurrence but trend of flow in the liquid phase.

It can be seen from flow field maps of Figs. 8(a) and 8(b) that flow velocities at top and bottom parts are quicker because solidification first happens from the bottom and solidification shrinkage is compensated for from the liquid surface. We can also find that velocities of flow at center zones of flow fields are quicker. Flow field can affect temperature field distributing. The isothermal zones are obviously stretched along liquid flow direction (Fig. 9); the change

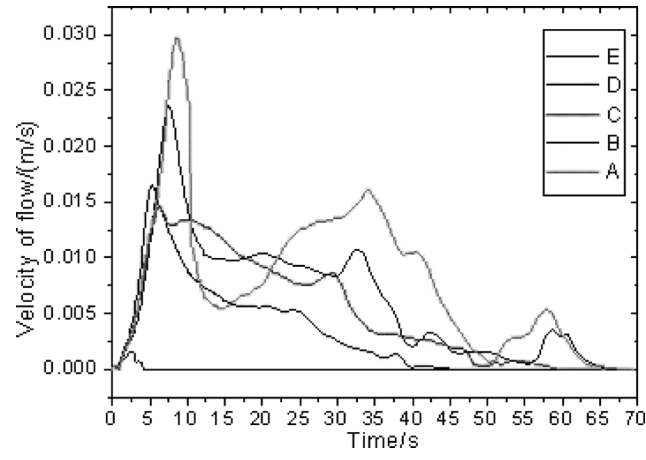

Fig. 11. Velocity of flow curves at locations of A, B, C, D and E.

trends of the isotherm are consistent with flow velocities in Fig. 9(b). We can find that temperature field distributes along the order of solidification regularly when there is not liquid flow (Fig. 10), and its isothermal zones are narrower than that in the presence of liquid flow. So, convection plays an importance role in even temperature fields. Hence convection can affect microstructure of the casting. It can be seen from Fig. 11 that the varieties of flow velocity at locations $\mathrm{A}, \mathrm{B}$, and $\mathrm{C}$ at initial stages increase first because of buoyancy-driven melt convection, and then decrease gradually due to the enhancement of 9SMn28 alloy viscosity. With the development of solidification, flow velocities at locations $\mathrm{A}, \mathrm{B}$, and $\mathrm{C}$ increase again owing to the function of feeding flows, flow velocities stop growing when solidification of these locations starts. Here, the melt becomes the mushy zone, and can be modeled by flow through porous media. Flow velocities at locations A, B, and C decrease gradually as the result of continuous increase of melt viscosity at final stages of solidification. The change degree of flow velocities from $\mathrm{A}$ to $\mathrm{B}$ and $\mathrm{C}$ is weakened gradually. The varieties of flow velocities at locations $\mathrm{D}$ and $\mathrm{E}$ increase first, and then decrease step by step. The peaks of flow velocities from $\mathrm{A}$ to $\mathrm{B}, \mathrm{C}, \mathrm{D}$, and $\mathrm{E}$ become smaller, and the arrived time of the peaks is increased by degrees.

\subsection{The Simulated Porosity Results and Discussion}

An isothermal curve method is applied to simulate porosity. It is the solid fraction isolines that the isothermal curves are denoted by solidus temperature. When the solid fractions arrive at such critical value $f_{s c}$ before solidification finish, macro-flows of the mushy zone are stopped, feeding is impossible. Therefore, a method of critical solid fraction can be applied to simulate porosity, i.e. the isothermal 

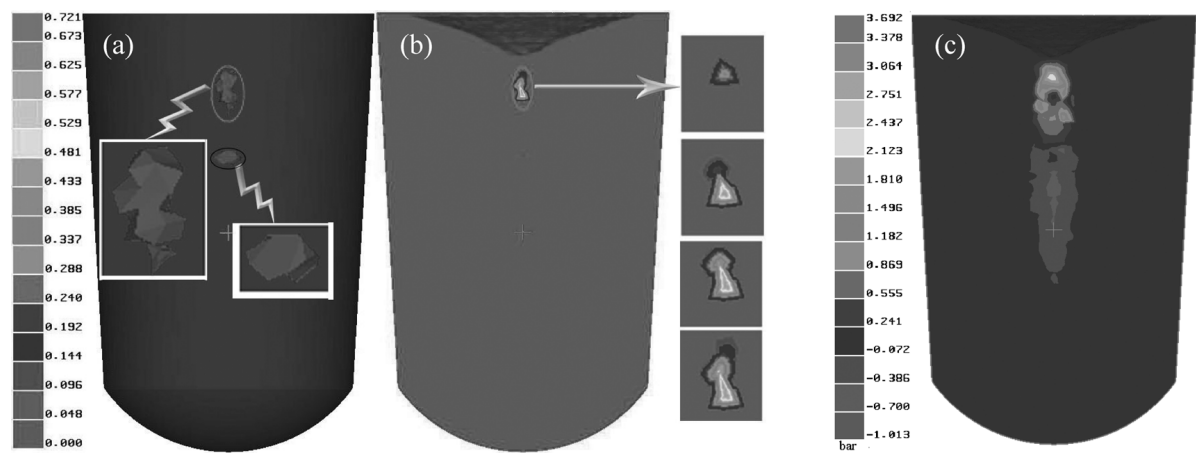

Fig. 12. The simulated porosity results and pressure change of the casting, (a) integer schematics of porosity, (b) the porosity in casting center surface, (c) pressure change. The left markings of the Fig. 12(a) and (c) are fraction porosity and pressure, respectively.

curve method. In this simulation, critical solid fraction $f_{s c}$ is $70 \%$. This paper ignores the influence of the gas owing to in the absence of volatile solute elements and tiny dissolved gases.

When the casting solidifies, pockets of liquid are created, surrounded by a mushy zone and then a solid shell. Automatically, the casting is divided into "regions" within which the fraction of solid is lower than one or those are bounded by walls (or symmetry planes). As solidification proceeds and depending upon the complexity of the geometry, the number of "regions" may increase with time. A region can thus be split in more regions. A region can disappear when all nodes have completely solidified. When a "region" is cooling down, some shrinkage occurs because the density is increasing with decreasing temperature. At each timestep, the accumulated shrinkage occurring at all the nodes which have a solid fraction equal to or lower than $f_{s c}$, plus those nodes between the $f_{s c}$ and $f_{s c}+$ feeding length isosurfaces (at which macroporosity can occur, beyond the $f_{s c}$ isosurface) is computed. This shrinkage is then distributed according to the different scenarios.

It can be seen from Figs. 12(a) and 12(b) that the simulated result of pipe shrinkage is consistent with that of this experiment (Fig. 14(a)). When temperature of casting surface reduces to freezing point, surface layers of the casting rapidly solidify under water cooling condition, a solidification layer is formed, such as marginal areas of the top surface of Fig. 12(b) and Fig. 14(a). At the beginning of solidification, solidification shrinkages happen, and solidification layers start to solid state shrinkages. Here, liquid state shrinkages still carry through within the casting; there is a depressed current on liquid surface. When solid state shrinkages are neither more nor less than equal to the summation of solidification shrinkages of solidification layers and liquid state shrinkages, the liquid surface shall not be gone down. When solid state shrinkages are less than the summation of solidification shrinkages of solidification layers and liquid state shrinkages, the liquid surface shall be gone down continually. With the development of solidification layers ceaselessly, the remainder of the liquid surface descends unceasingly, and cross-section is reduced. A big end down pipe shrinkage is formed in the casting, such as the top surface of Fig. 12(b) and Fig. 14(a).

The simulated result of porosity is identical with that of the experiment basically. Porosity that is formed and trapped in the mushy zone of solidifying alloys is the result

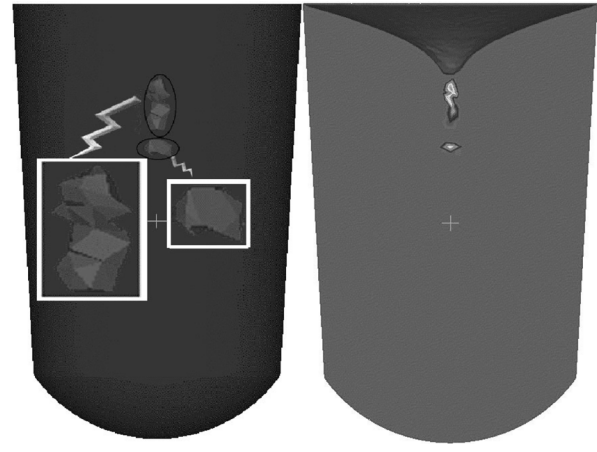

Fig. 13. The simulated porosity results of no liquid flow.

of concomitant mechanism, solidification shrinkage, which induces a negative volume variation during the phase transformation of 9SMn28 alloy, has to be compensated for by interdendritic liquid flow (e.g., feeding) to avoid porosity. The porosity might start to form in a partially closed liquid region when this liquid region is closed. The complete growth of porosity, from the instant of heterogeneous nucleation until the last growth stage, is along the order of solidification (Fig. 12(b)). Feeding induces a pressure drop in the mushy zone (Fig. 12(c)). ${ }^{26}$ The pressure drop is calculated using the following equation: $\Delta x / \Delta P=K /(\mu v)$. It can be found from Fig. 13 that pipe shrinkage is bigger, the top surface is more smoothness, the porosity is smaller, and the porosity morphology is more regulation under no liquid flow condition.

\subsection{The Simulated Microstructure Results and Dis- cussion}

The simulated and experimental microstructure results of the casting are shown in Fig. 14, and the different gray scales represent different crystallographic orientations.

It can be seen from Figs. 14(a) and 14(b) that the simulated results are consistent with that of the experiment. The rate of columnar grains to equiaxed grains is same as the casting, the grains of columnar-to-equiaxed transition (CET) zone are both larger, and the grains of equiaxed zone are both fine and uniformity. There are not grains in the locations of pipe shrinkage; buoyancy-driven melt convection can significantly stabilize the planar morphology, so, the CET is complanate. However, the locations of the simulated porosity are not full solid; the simulated microstructure results can not realize their simulation.

Crystallization process conforms to the actual situation. 


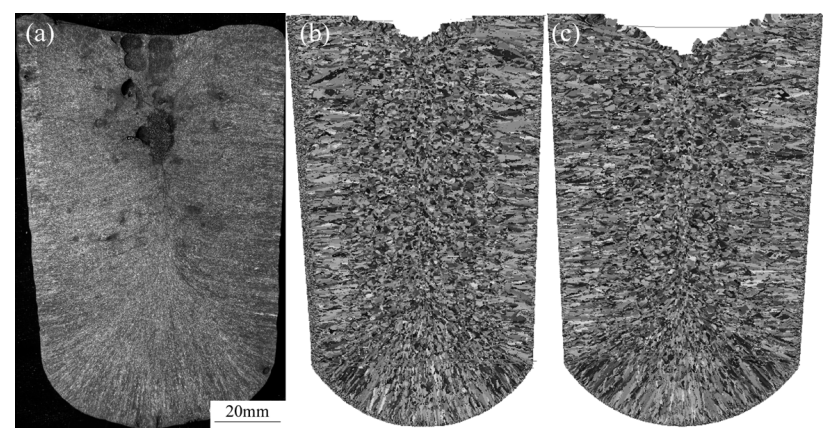

Fig. 14. The experimental (a) and simulated (b) microstructure results of the casting and simulated results of no liquid flow (c).

Among the many grains formed at external surface of the casting only a few long columnar grains succeed in growing. In this columnar zone, a few grains have clearly nucleated in the bulk of the liquid ahead of the growing columnar front. If they have the proper orientation, they can grow and become elongated. The thermal gradient decreases, and the undercooling liquid region ahead of the columnar dendrite tips becomes wider. Thus, the density of the grains nucleated in the bulk of the liquid increases and their growth finally stops the columnar front. These grains, however, still have an elongated shape because of the thermal gradient. As the thermal gradient continues to decrease in the remaining liquid, such grains finally become truly equixed. ${ }^{9)}$ Columnar grains are greater under no liquid flow condition (Fig. 14(c)).

\section{Conclusions}

(1) The casting of 9SMn28 alloy is a stepwise solidification mode under water cooling condition.

(2) Convection is mainly buoyancy-driven flows and feeding flows in perpendicular direction of the solid-liquid interface under water cooling condition. Buoyancy-driven melt convection comes into being owing to gravity; feeding flows are formed by solidification shrinkage. Liquid flows are the reason of shaggy on free surface; it can decrease pipe shrinkage and columnar grain zone. Flow field plays an importance role in even temperature field and irregulation porosity morphology.

(3) The simulation result of pipe shrinkage is consistent with that of this experiment; it is formed because solid state shrinkages are less than the summation of solidification shrinkages of solidification layers and liquid state shrinkages, it results in the liquid surface of the casting fall continually. The simulation result of porosity is identical with this experiment basically; the porosity might start to form in a partially closed liquid region when this liquid region is closed.

(4) The simulation results of microstructure in free-cutting steel 9SMn28 are consistent with that of this experiment with convection and porosity. Crystallization process conforms to the actual situation.

\section{Acknowledgements}

The authors would like to be grateful for the National Natural Science Fund (50874007) for their financial support and ESI group for providing Procast Software.

\section{REFERENCES}

1) D. G. Eskin, R. Nadella and L. Katgerman: Acta Mater., 56 (2008), 1358.

2) C. W. Lan and C. Y. Tu: J. Cryst. Growth, 220 (2000), 619.

3) C. W. Lan, Y. W. Yang and H. Z. Chen: Metall. Mater. Trans. A, 33A (2002), 3011.

4) C. W. Lan, M. H. Lee, M. H. Chuang and C. J. Shih: J. Cryst. Growth, 295 (2006), 202.

5) D. Li, R. Li and P. Zhang: Appl. Math. Model., 31 (2007), 971.

6) Y. H. Shin and C. P. Hong: ISIJ Int., 42 (2002), 359.

7) Ch. Pequet, M. Gremaud and M. Rappaz: Metall. Mater. Trans. A, 33A (2002), 2095.

8) M. Rappaz and Ch.-A. Gandin: Acta Metall. Mater., 41 (1993), 345.

9) Ch.-A. Gandin and M. Rappaz: Acta Metall. Mater., 42 (1994), 2233.

10) L. Nastac and D. M. Stefanescu: Model. Simul. Mater. Sci. Eng., 5 (1997), 391.

11) M. F. Zhu, J. M. Kim and C. P. Hong: ISIJ Int., 41 (2001), 436

12) M. F. Zhu and C. P. Hong: ISIJ Int., 41 (2001), 992.

13) H. L. Lukas, J. Weiss and E. Th. Henig: Calphad, 6 (1982), 229

14) U. R. Kattner: JOM, 49 (1997), 14

15) N. Saunders and A. P. Miodownik: Pergamon Materials Series, 1 (1998), 33.

16) Ph. Thévoz, J. L. Desbiolles and M. Rappaz: Metall. Mater. Trans. A, 20A (1989), 311.

17) T. U. Kämpfer: Modeling of Macrosegregation Using an Adaptive Domain Decomposition Method, Lausanne: EPFL, (2002), 13.

$18)$ W. D. Bennon and F. P. Incropera: Metall. Mater. Trans. B, 18B (1987), 611.

19) S. D. Felicelli, J. C. Heinrich and D. R. Poirier: Metall. Mater. Trans. $B, 22 B$ (1991), 847.

20) R. Mehrabian, M. Keane and M. C. Flemings: Metall. Mater. Trans. B, 2B (1971), 1209.

21) O. Nielsen, L. Arnberg, A. Mo and H. Thevik: Metall. Mater. Trans. A, 30A (1999), 2455.

22) W. Kur, B. Giovanolab and R. Trivedi: Acta Metall. Mater, 34 (1986), 82

23) Z. Ignaszak, M. Hajkowski and J. Hajkowski: J. Mater. Sci., 12 (2006), 124

24) Ch.-A. Gandin and M. Rappaz: Acta Metall. Mater., 45 (1997), 2187.

25) M. Wu, A. Ludwig, A. Bührig-Polaczek, M. Fehlbier and P. R. Sahm: Int. J. Heat Mass Transfer, 46 (2003), 2819.

26) G. Couturier and M. Rappaz: Model. Simul. Mater. Sci. Eng., 14 (2006), 253. 